\title{
Coherent Quasiclassical Dynamics of a Persistent Current Qubit
}

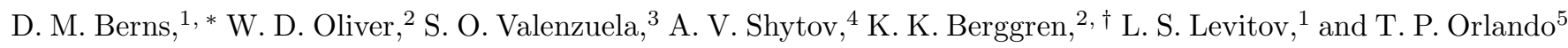 \\ ${ }^{1}$ Department of Physics, Massachusetts Institute of Technology, Cambridge MA 02139 \\ ${ }^{2}$ MIT Lincoln Laboratory, 244 Wood Street, Lexington, MA 02420 \\ ${ }^{3}$ MIT Francis Bitter Magnet Laboratory, Cambridge, MA 02139 \\ ${ }^{4}$ Physics Department, Brookhaven National Laboratory, Upton, NY 11973-5000 \\ ${ }^{5}$ Department of Electrical Engineering and Computer Science, \\ Massachusetts Institute of Technology, Cambridge, MA 02139
}

\begin{abstract}
A new regime of coherent quantum dynamics of a qubit is realized at low driving frequencies in the strong driving limit. Coherent transitions between qubit states occur via the Landau-Zener process when the system is swept through an energy-level avoided crossing. The quantum interference mediated by repeated transitions gives rise to an oscillatory dependence of the qubit population on the driving field amplitude and flux detuning. These interference fringes, which at high frequencies consist of individual multiphoton resonances, persist even for driving frequencies smaller than the decoherence rate, where individual resonances are no longer distinguishable. A theoretical model that incorporates dephasing agrees well with the observations.
\end{abstract}

PACS numbers: 03.67.Lx,03.65.Yz,85.25.Cp,85.25.Dq

Macroscopic quantum systems coherently driven by external fields provide new insights into the fundamentals of quantum mechanics and hold promise for applications such as quantum computing [1]. Superconducting Josephson devices are model quantum systems that can be manipulated by RF driving fields [2], and recent years have seen rapid progress in the understanding of their quantum dynamics 3, 4, 5, 6, 7, 8, 9, 10]. Quantum coherence of these systems can be probed by temporal Rabi oscillations [3, 6, 7, 8, 9, 10]. There, the drivingfield frequency $\nu$ equals the energy level separation $\Delta E$, and the population of the two levels oscillates at a frequency $\omega_{\mathrm{R}}$ much smaller than $\Delta E$. In the weak driving limit, $\hbar \omega_{\mathrm{R}} \approx A \ll \Delta E=h \nu$, where $A$ is the driving amplitude parameterized in units of energy.

Coherent quantum dynamics can also be investigated at driving frequencies much less than $\Delta E$, and at strong driving amplitude $A \approx \Delta E \gg h \nu$. In this case, the transitions occur via the Landau-Zener (LZ) process at a level crossing 11, 12]. Acting as a coherent beamsplitter, LZ transitions create a quantum superposition of the ground and excited states and, upon repetition, induce quantum mechanical interference. The latter leads to Stueckelberg-type oscillations [13, 14] in analogy to a Mach-Zehnder (MZ) interferometer [15, 16]. These oscillations are also related to photoassisted transport 17, 18, 19 and Rabi oscillations observed in the multiphoton regime 6, 20]. MZ-type interference is a unique signature of temporal coherence complementary to Rabi oscillations, with the time between sequential LZ transitions clocking the dynamics similarly to Rabi pulse width.

In this Letter, we report a new quasiclassical regime which exhibits coherence even at driving frequencies low compared to dephasing rate, $\nu T_{2} \lesssim 1$ 21]. This occurs because the interval between consecutive LZ transitions,

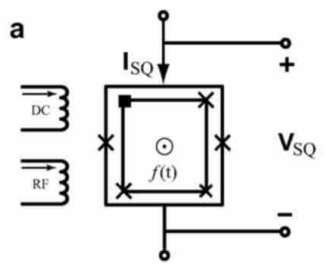

b

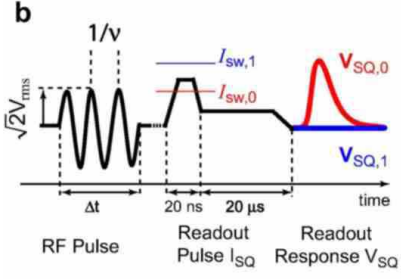

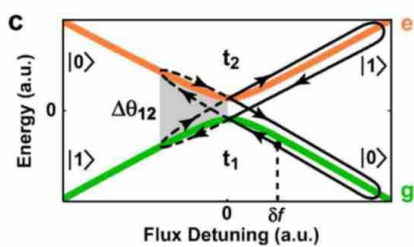

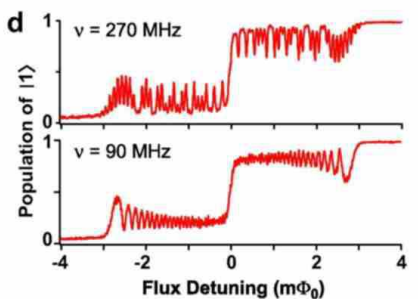

FIG. 1: (a) Schematic of the PC qubit surrounded by a DC SQUID readout. DC and RF fields control the state of the qubit. (b) An RF pulse of duration $\Delta t \gg \nu^{-1}$ drives the qubit, and its state is inferred from the voltage $V_{\mathrm{SQ}}$ across the SQUID pulsed with current $I_{\mathrm{SQ}}$. (c) The qubit experiences two Landau-Zener transitions over a single RF period, accumulating a relative phase $\Delta \theta_{12}$ between them. (d) The resulting interference fringes in qubit population for $\nu=270$ and $90 \mathrm{MHz}$, and $V_{\mathrm{rms}}=240$ and $171 \mathrm{mV}$ respectively (vertical lines on Fig 2).

relevant for MZ interference, is only a fraction of the driving field period. We investigate the crossover between the multiphoton and quasiclassical regimes, demonstrating that coherent MZ-type interference fringes in the qubit population persist for frequencies $\nu T_{2} \lesssim 1$ even though individual multiphoton resonances can no longer be resolved. This behavior should be contrasted with Rabi oscillations, where at low driving frequency, $\nu T_{2} \lesssim 1$, there is no signature of coherence. The crossover between the two regimes, $\nu T_{2} \sim 1$, is also influenced by inhomogeneous broadening, as discussed below.

In our experiment we utilize a persistent-current (PC) 
qubit 22]: a superconducting loop interrupted by three Josephson junctions (JJ), one of which has a reduced cross-sectional area (Fig [ $\mathrm{H}$ ). A time-dependent magnetic flux $f(t)=f^{\mathrm{dc}}+f^{\mathrm{ac}}$ controls the qubit. For $f(t) \approx \Phi_{0} / 2$, the qubit exhibits a double-well potential profile with individual wells representing diabatic circulating-current states, $|0\rangle$ and $|1\rangle$, with energies $\pm \epsilon \propto$ $\pm \delta f$, where $\delta f \equiv f^{\mathrm{dc}}-\Phi_{0} / 2$ is the flux detuning. These states are coupled with a tunneling energy $\Delta$. The driving and readout pulse sequence is illustrated in Fig (b). Qubit transitions are driven by a microwave flux $f^{\text {ac }} \propto A \cos 2 \pi \nu t$, with $A$, parameterized in units of energy, proportional to the microwave source voltage $V_{\text {rms }}$. The qubit state is read out with a DC SQUID, whose switching current $I_{\mathrm{SW}}$ depends on the flux generated by the qubit and, thereby, the qubit circulatingcurrent state. The device was fabricated at Lincoln Laboratory using a fully-planarized niobium trilayer process and optical lithography. The device has a critical current density $J_{\mathrm{c}} \approx 160 \mathrm{~A} / \mathrm{cm}^{2}$, and the characteristic Josephson and charging energies are $E_{\mathrm{J}} \approx(2 \pi \hbar) 300 \mathrm{GHz}$ and $E_{\mathrm{C}} \approx(2 \pi \hbar) 0.65 \mathrm{GHz}$ respectively. The ratio of the qubit JJ areas is $\alpha \approx 0.84$, and $\Delta \approx(2 \pi \hbar) 10 \mathrm{MHz}$. The experiments were performed in a dilution refrigerator at a base temperature of $20 \mathrm{mK}$. The device was magnetically shielded, and all electrical lines were carefully filtered and attenuated to reduce noise (see Ref. 15] for details).

The qubit dynamics in the strongly driven limit is influenced by quantum interference at sequential LZ transitions. As illustrated in Fig प(c), the qubit is initially prepared in the ground state at flux detuning $\delta f$, and, after a first LZ transition at time $t_{1}$, it is in a coherent superposition of the two diabatic states. For times $t_{1}<t<t_{2}$, the superposition state accumulates a relative phase $\Delta \theta_{12}$, which mediates the quantum interference at the second LZ transition at time $t_{2}$. The sequence of two LZ transitions, repeated many times during the RF pulse, is analogous to a cascade of MZ interferometers. One expects MZ-type interference fringes in the qubit population due to changes in $\Delta \theta_{12}$ associated with changes in $V_{\mathrm{rms}}$ and $\delta f$, which are indeed observed (Fig 1).

Figure 2 presents the measured qubit population of state $|1\rangle$ (color scale) as a function of $V_{\mathrm{rms}}$ and $\delta f$ for high- and low-frequency driving, $\nu=270$ and $90 \mathrm{MHz}$ respectively. Population transfer due to qubit driving appears at $V_{\text {rms }}$ exceeding a threshold value which varies linearly with $|\delta f|$ and symmetrically about the qubit step. For high-frequency driving, $\nu T_{2} \gtrsim 1$, the individual multiphoton resonances are distinguishable and form a "Bessel ladder" 15](Fig. 22). The population of state $|1\rangle$ for the $n^{\text {th }}$-photon resonance follows a Bessel-function dependence, $J_{n}^{2}(A / h \nu)$. The range of $\delta f$ in Fig. 2a accommodates photon transitions with $n=1-45$, which together define coherent MZ interference-fringe bands of discrete resonances.

In contrast, for low-frequency driving, $\nu T_{2} \lesssim 1$, the in-

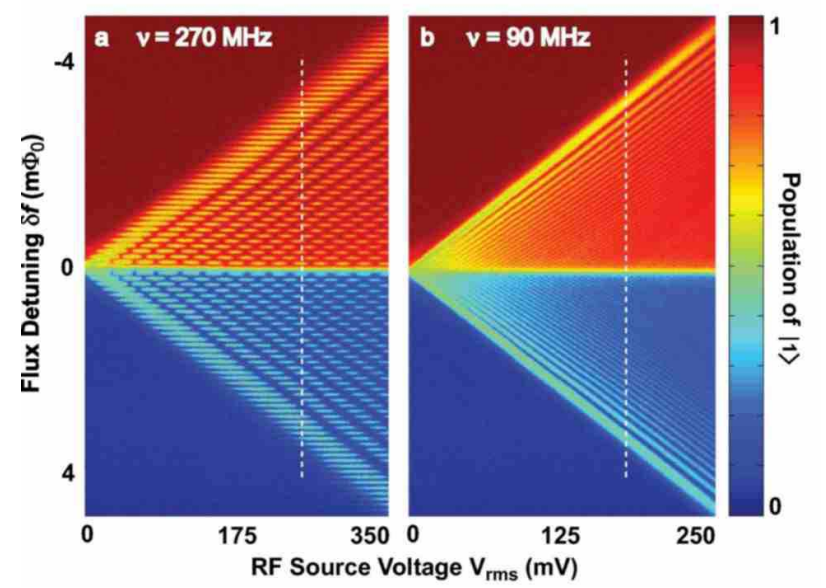

FIG. 2: Measured qubit population at strong driving in two regimes. (a) $\nu=270 \mathrm{MHz}$. Multiphoton resonances of order up to $n=45$ can be discerned $\left(\nu T_{2}>1\right)$. (b) $\nu=90 \mathrm{MHz}$. Individual resonances are no longer distinguishable $\left(\nu T_{2} \lesssim\right.$ $1)$, but coherent interference is still observed. Vertical lines indicate the scans displayed in Fig[1]. A pulse of duration $\Delta t=3 \mu \mathrm{s}$ was used in both cases.

dividual photon resonances are no longer distinguishable because the resonance widths exceed the resonance spacing (Fig. 2b). Nonetheless, the MZ interference-fringe bands, a signature of coherence in the strongly driven regime, indicate that the coherent interference mediating the population transfer persists.

To understand these results we consider a driven qubit subject to the effects of decoherence $(\hbar=1)$ :

$$
\mathcal{H}=-\frac{1}{2}\left(\begin{array}{cc}
h(t) & \Delta \\
\Delta & -h(t)
\end{array}\right)
$$

Here, $h(t)=\epsilon+\delta \epsilon(t)+A \cos 2 \pi \nu t$ is the energy detuning from an avoided crossing modulated by the driving field in the presence of classical noise $\delta \epsilon(t)$. By a gauge transformation, the Hamiltonian is brought to the form

$$
\mathcal{H}=-\frac{1}{2}\left(\begin{array}{cc}
0 & \Delta(t) \\
\Delta^{*}(t) & 0
\end{array}\right), \quad \Delta(t)=\Delta e^{-i \phi(t)},
$$

where $\phi(t)=\int_{0}^{t} h(\tau) d \tau$. Perturbation theory gives the rate of LZ transitions between the states $|0\rangle$ and $|1\rangle$ :

$$
W=\lim _{\delta t \rightarrow \infty}\left|A_{t, t^{\prime}}\right|^{2} / \delta t, \quad A_{t, t^{\prime}}=\int_{t}^{t^{\prime}} \Delta\left(t^{\prime}\right) d t^{\prime},
$$

where $\delta t=t^{\prime}-t>0$, and the limit physically means that $\delta t$ is large compared to $T_{2}$.

For the perturbation approach to be valid, the change of qubit population must be slow on the scale of $T_{2}$. This condition can be written down as $W \ll \Gamma_{2}=1 / T_{2}$. We stress that this inequality does not imply that the effect of driving the qubit is weak. The rate $W$ can still be large compared to the inelastic relaxation rate $\Gamma_{1}$, leading to the strong deviation of population from equilibrium observed in our experiment. 
To evaluate $W$, we write the expression in Eq.(36) as

$$
W=\frac{1}{4} \int\left\langle\Delta(t+\tau) \Delta^{*}(t)\right\rangle d \tau, \quad \Delta(t)=\Delta e^{-i \phi(t)} .
$$

By introducing Bessel functions in the Fourier series of $e^{-i(A / \omega) \sin \omega t}, \omega=2 \pi \nu$, we have

$$
e^{-i \phi(t)}=e^{-i \epsilon t-i \delta \phi(t)} \sum_{n} J_{n}(x) e^{i \omega n t}, \quad x=\frac{A}{\omega}=\frac{A}{h \nu} .
$$

We average over $\delta \phi(t)$ with the help of the white noise model $\left\langle e^{i \delta \phi(t)-i \delta \phi\left(t^{\prime}\right)}\right\rangle=e^{-\Gamma_{2}\left|t-t^{\prime}\right|}$, and integrate in (4) as $\int e^{-i(\epsilon-\omega n) \tau-\Gamma_{2}|\tau|} d \tau=2 \Gamma_{2} /\left((\epsilon-\omega n)^{2}+\Gamma_{2}^{2}\right)$ to obtain

$$
W(\epsilon, A)=\frac{\Delta^{2}}{2} \sum_{n} \frac{\Gamma_{2} J_{n}^{2}(x)}{(\epsilon-\omega n)^{2}+\Gamma_{2}^{2}} .
$$

For large $n$, Bessel functions can be expressed through the Airy function $\operatorname{Ai}(u)=\frac{1}{\pi} \int_{0}^{\infty} \cos \left(u y+\frac{1}{3} y^{3}\right) d y$ as $J_{n}(x)=a \operatorname{Ai}(a(n-x)), a=(2 / x)^{1 / 3}$. Using the identity $\cot z=\sum(z-\pi n)^{-1}$ we approximate Eq. (5) as

$$
W=\frac{\pi a^{2} \Delta^{2}}{2 \omega} \operatorname{Im} \cot \left(\frac{\pi}{\omega}\left(\epsilon-i \Gamma_{2}\right)\right) \operatorname{Ai}^{2}\left(\frac{a}{\omega}(\epsilon-A)\right) .
$$

There are two main regimes exhibited by this expression: (i) $\nu \gtrsim \Gamma_{2}$, and (ii) $\nu \lesssim \Gamma_{2}$. In case (i), we have a sum of non-overlapping resonances. For each value of $\epsilon$, the sum is dominated by the term with $n$ the nearest integer to $\epsilon / \omega$, giving rise to resonances of strength $J_{n}^{2}(x)$, the Bessel ladder of Ref. [15].

In contrast, in case (ii), the peaks in Eq. (5D) are overlapping. Setting cot $=i$ in Eq.(6) [21], we obtain

$$
W(\epsilon, A) \approx \frac{\pi a^{2} \Delta^{2}}{2 \omega} \operatorname{Ai}^{2}(a(\epsilon-A) / \omega) .
$$

The effect of $\Gamma_{2}$ on the Airy function oscillation is small at $\Gamma_{2} \lesssim(2 \pi / a) \nu$. Since $a \approx 0.3$ for $\epsilon / h \nu \lesssim 50$, this condition is compatible with $\nu \lesssim \Gamma_{2}$. Eq.(7) can also be obtained by considering just two subsequent passages of a level crossing at a short time separation $\left|t_{2}-t_{1}\right| \ll \nu^{-1}$, and ignoring the periodicity of the driving.

Since $\operatorname{Ai}(u<0)$ oscillates as $\pi^{-1 / 2}|u|^{-1 / 4} \cos \left(\frac{2}{3}|u|^{3 / 2}-\right.$ $\left.\frac{\pi}{4}\right)$, while $\operatorname{Ai}(u>0)$ decays exponentially, Eq.(7) implies that the transitions occur only for $A \gtrsim \epsilon$, with a rate which oscillates as a function of $A-\epsilon$. The oscillations are the same for both integer and noninteger $\epsilon / h \nu$, confirming that, while the resonances merge into a continuous band, the interference fringes persist at $\nu \lesssim \Gamma_{2}$, in agreement with our observations.

To describe the population dynamics in the presence of driving, we employ a rate equation approach, in which the qubit level occupations obey $\dot{p}_{i}=\sum_{j} g_{i j} p_{j}$, where

$$
g_{01}=-g_{11}=W+\Gamma_{1}, \quad g_{10}=-g_{00}=W+\Gamma_{1}^{\prime} .
$$

Here, $\Gamma_{1}=1 / T_{1}, \Gamma_{1}^{\prime}=\Gamma_{1} e^{-\beta \epsilon}$ are the down and up relaxation rates. The magnetization of the stationary

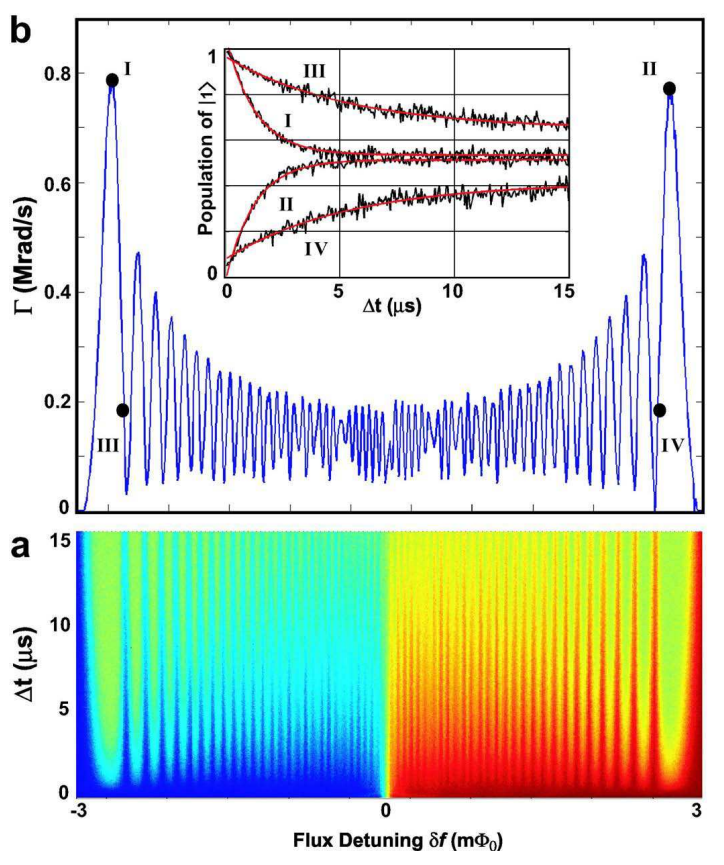

FIG. 3: (a) Time evolution of excited state population $(\nu=$ $90 \mathrm{MHz}, V_{r m s}=171 \mathrm{mV}$ ) obtained by varying the pulse width $\Delta t$. (b) The characteristic rate $\Gamma$ as a function of flux detuning $\delta f$ obtained by fitting to the exponential time dependence [Eq.(9)] (inset shows examples of fits for the points I, II, III and IV).

state is $m_{s}=p_{0}-p_{1}=\left(\Gamma_{1}-\Gamma_{1}^{\prime}\right) /\left(2 W+\Gamma_{1}+\Gamma_{1}^{\prime}\right)$, which gives the equilibrium value $m_{0}=\tanh \frac{1}{2} \beta \epsilon$ at weak excitation, and $m_{s} \ll m_{0}$ at high excitation.

To validate this model, we investigate the interference fringes in the excited state population as a function of $\mathrm{RF}$ pulse length $\Delta t(\mathrm{Fig}[\mathrm{B})$. The rate equation predicts an exponential time dependence for the magnetization,

$$
m(\Delta t)=m_{s}+\left(m_{0}-m_{s}\right) e^{-\Gamma \Delta t}, \quad \Gamma=2 W+\Gamma_{1}+\Gamma_{1}^{\prime} .
$$

By fitting exponentials [Eq.(91] to the qubit population at each flux detuning, we find the rate $\Gamma$ which characterizes how fast the stationary state is approached (Fig [3). Since our $T_{1} \approx 20 \mu \mathrm{s}[15$ is much longer than the observed transition time, we have $\Gamma \approx 2 W$. Comparing the extracted $\Gamma$ with Eq.(7), we obtain $\Delta / 2 \pi \hbar=13 \mathrm{MHz}$.

In Fig. 4 we compare the experimental characteristic rate and qubit population with those predicted by the model [Eqs.(5), (9)]. From the best fits we obtain $\Gamma_{2} / 2 \pi=12-18 \mathrm{MHz}\left(T_{2} \approx 9-13 \mathrm{~ns}\right)$, consistent with the transition between the multiphoton and quasiclassical regimes of Fig. 22 $270 \mathrm{MHz}>\Gamma_{2}=1 / T_{2} \gtrsim 90 \mathrm{MHz}$.

Inhomogeneous broadening is incorporated into the model by assuming a Gaussian broadening mechanism with standard deviation $\sigma / 2 \pi=40-45 \mathrm{MHz}$. The resulting power-broadened linewidth is approximately $150 \mathrm{MHz}$, consistent with the linewidth observed in

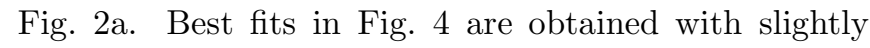
different values of $\Gamma_{2}$ and $\sigma$ within the ranges above. By 

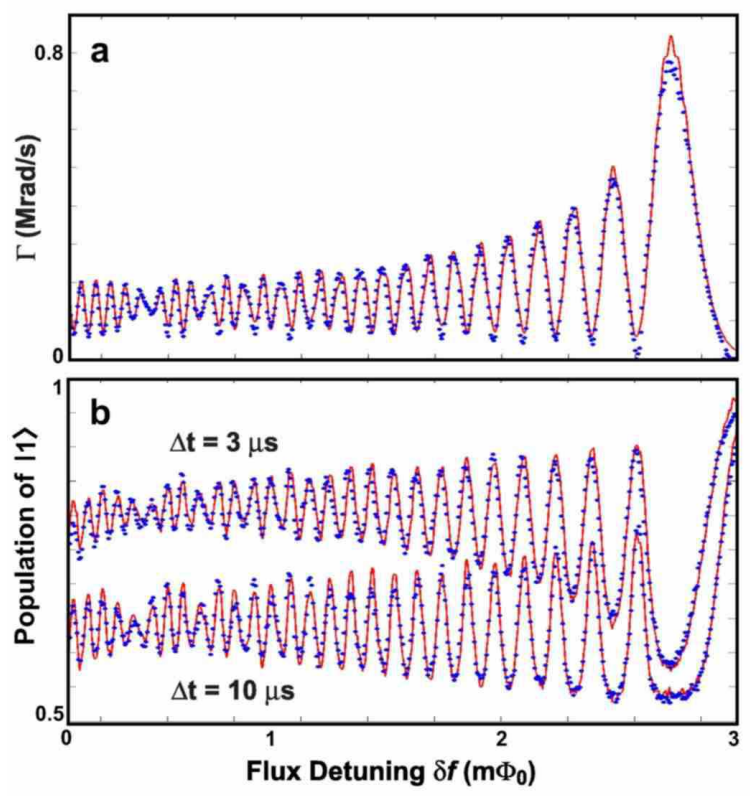

FIG. 4: Comparison of experiment (blue) and theory (red). (a) The transition rate from the right half of Fig 3] fitted with $\Gamma$ defined by Eqs. (5), (9). (b) State $|1\rangle$ occupation taken from Fig[3], compared to the model, Eq. (9].

using the fit parameters for the $3 \mu s$ magnetization curve, we can calculate the qubit population in the multiphoton (Fig. 5 $\mathrm{k}$ ) and quasiclassical (Fig. 5 5 ) regimes as a function of $\delta f$ and $V_{\text {rms }}$.

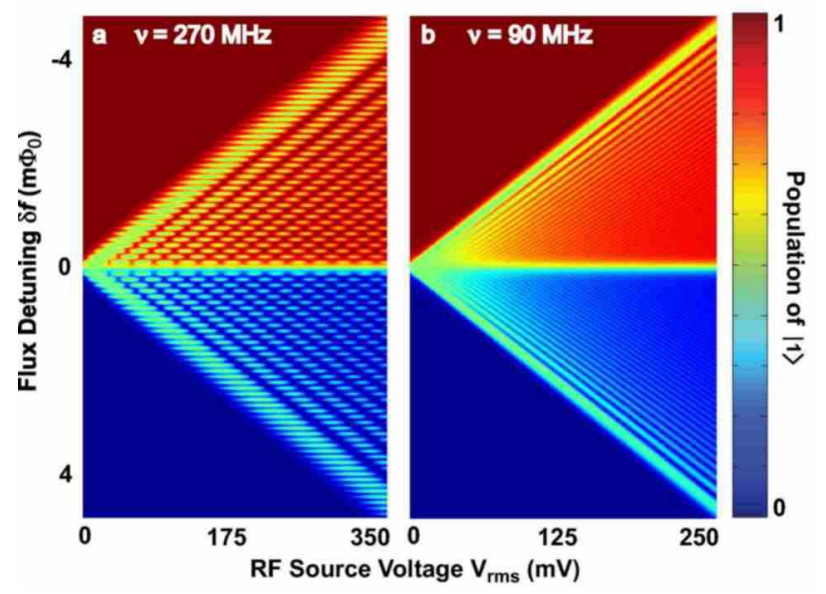

FIG. 5: Simulation of qubit population using model parameters extracted from data. (a) $\nu=270 \mathrm{MHz}$. (b) $\nu=90 \mathrm{MHz}$.

In conclusion, we have observed quantum coherent qubit dynamics at strong driving for frequencies smaller than the dephasing rate. In this limit, well-resolved multiphoton transitions merge into a continuous band, while the Mach-Zehnder-like coherent interference pattern persists. A simple model of a driven two-level system subject to decoherence is in remarkable agreement with the observed interference patterns.

We thank V. Bolkhovsky, G. Fitch, D. Landers, E. Macedo, R. Slattery, and T. Weir at MIT Lincoln Laboratory for fabrication and technical assistance; D. Cory, A. J. Kerman, and S. Lloyd for helpful discussions. This work was supported by AFOSR (F49620-01-1-0457) under the DURINT program. The work at Lincoln Laboratory was sponsored by the US DoD under Air Force Contract No. FA8721-05-C-0002. AVS acknowledges support by US DOE under contract No. DEAC 02-98 CH 10886.

* Electronic address: dmb@mit.edu

$\dagger$ Present address: EECS Department, MIT

[1] J. E. Mooij, Science 307, 1210 (2005).

[2] Y. Makhlin, G. Schön, A. Shnirman, Rev. Mod. Phys. 73, 357 (2001).

[3] Y. Nakamura, Y. A. Pashkin, J. S. Tsai, Nature 398, 786 (1999).

[4] J. R. Friedman, V. Patel, W. Chen, S. K. Tolpygo, J. E. Lukens, Nature 406, 43 (2000).

[5] C. H. van der Wal, A. C. J. ter Haar, F. K. Wilhelm, R. N. Schouten, C. J. P. M. Harmans, T. P. Orlando, S. Lloyd, J. E. Mooij, Science 290, 773 (2000).

[6] Y. Nakamura, Y. A. Pashkin, J. S. Tsai, Phys. Rev. Lett. 87, 246601 (2001).

[7] D. Vion, A. Aassime, A. Cottet, P. Joyez, H. Pothier, C. Urbina, D. Esteve, and M. H. Devoret, Science 296, 886 (2002).

[8] Y. Yu, S. Han, X. Chu, S.-I. Chu, Z. Wang, Science 296, 889 (2002).

[9] J. M. Martinis, S. Nam, J. Aumentado, C. Urbina, Phys. Rev. Lett. 89, 117901 (2002).

[10] I. Chiorescu, Y. Nakamura, C. J. P. M. Harmans, J. E. Mooij, Science 299, 1869 (2003).

[11] A. V. Shytov, D. A. Ivanov, M. V. Feigel'man, Eur. Phys. J. B 36, 263 (2003).

[12] A. Izmalkov, M. Grajcar, E. Il'ichev, N. Oukhanski, Th. Wagner, H.-G. Meyer, W. Krech, M. H. S. Amin, A. Maassen van den Brink and A. M. Zagoskin, Europhys. Lett. 65, 844 (2004).

[13] E. C. G. Stueckelberg, Helv. Phys. Acta 5, 369 (1932).

[14] H. Nakamura, Nonadiabatic Transition (London, England: World Scientific, 2001).

[15] W. D. Oliver, Y. Yu, J. C. Lee, K. K. Berggren, L. S. Levitov, T. P. Orlando, Science 310, 1653 (2005).

[16] M. Sillanpää, T. Lehtinen, A. Paila, Yu. Makhlin, P. Hakonen, Phys. Rev. Lett. 96, 187002 (2006).

[17] P. K. Tien and J. P. Gordon, Phys. Rev. 129, 647 (1963).

[18] L. P. Kouwenhoven, S. Jauhar, J. Orenstein, P. L. McEuen, Y. Nagamune, J. Motohisa, and H. Sakaki, Phys. Rev. Lett. 73, 3443 (1994).

[19] Y. Nakamura, J. S. Tsai, J. Supercond. 12, 799 (1999).

[20] S. Saito, T. Meno, M. Ueda, H. Tanaka, K. Semba, H. Takayanagi, Phys. Rev. Lett. 96, 107001 (2006)

[21] We emphasize that the crossover between high and low frequencies in this case occurs at $\nu T_{2} \sim 1$ rather than at $\omega T_{2} \sim 1$. This can be inferred from the expression $\operatorname{Im} \cot \left(\pi\left(\epsilon-i \Gamma_{2}\right) / \omega\right)$, Eq. [6], which describes multiphoton resonances broadening. At large $\Gamma_{2}$ this gives $1+2 e^{-2 \pi \Gamma_{2} / \omega} \cos (2 \pi \epsilon / \omega)+$ $O\left(e^{-4 \pi \Gamma_{2} / \omega}\right)$. Multiphoton oscillations become dephased at $\nu \approx \Gamma_{2}$.

[22] T. P. Orlando, J. E. Mooij, L. Tian, C. H. van der Wal, L. Levitov, S. Lloyd, and J. J. Mazo, Phys. Rev. B 60, 15398 (1999). 